\title{
VLBI observation of narrow bandwidth signals from the spacecraft
}

\author{
Fuyuhiko Kikuchi ${ }^{1}$, Yusuke Kono ${ }^{2}$, Makoto Yoshikawa ${ }^{3}$, Mamoru Sekido ${ }^{4}$, Masafumi Ohnishi' ${ }^{5}$, \\ Yasuhiro Murata ${ }^{3}$, Jinsong Ping ${ }^{6}$, Qinghui Liu ${ }^{6}$, Koji Matsumoto ${ }^{6}$, Kazuyoshi Asari ${ }^{6}$, \\ Seiitsu Tsuruta ${ }^{6}$, Hideo Hanada ${ }^{2}$, and Nobuyuki Kawano ${ }^{6}$ \\ ${ }^{1}$ Department of Astronomical Science, Graduate University for Advanced Studies, \\ 2-12 Hoshigaoka, Mizusawa, Iwate 023-0861, Japan \\ ${ }^{2}$ Division of Radio Astronomy, National Astronomical Observatory, 2-21-1 Osawa, Mitaka, Tokyo 181-8588, Japan \\ ${ }^{3}$ Institute of Space and Astronautical Science, Japan Aerospace Exploration Agency, \\ 3-1-1 Yoshinodai, Sagamihara, Kanagawa 229-8510, Japan \\ ${ }^{4}$ Kashima Space Reserch Center, National Institute of Information and Communications Technology, \\ 893-1 Hirai, Kashima, Ibaraki 314-8510, Japan \\ ${ }^{5}$ Fujitsu Limited, 1-9-3 Nakasen Mihama-ku, Chiba 261-8588, Japan \\ ${ }^{6}$ Division of Radio Astronomy, National Astronomical Observatory, 2-12 Hoshigaoka, Mizusawa, Iwate 023-0861, Japan
}

(Received May 7, 2004; Revised October 13, 2004; Accepted October 13, 2004)

\begin{abstract}
We carried out a series of VLBI observations of Nozomi by using a dedicated narrow bandwidth VLBI system. The three carrier waves with frequency interval of $515 \mathrm{kHz}$ were recorded in 3 channels of the system and correlated by a software method. As a result of the correlation, the residual fringe phases of the main carrier wave are obtained for every 1.3 seconds. We can also continuously track them for 100 minutes. The variation of the residual fringe phase is $+/-150$ degrees. Moreover, we can derive succesively the group delay for every 100 seconds by using these three carrier waves. The RMS of the group delays is 13 nsec and its average is well accorded with the delay determined by the range and Doppler measurements within an error of 2 nsec. Consequently, we confirmed the validity of the narrow bandwidth VLBI system, and it could be expected that this system, in addition to range and Doppler measurements, can be applied to three-dimensional tracking of a spacecraft and the precise gravity measurement of the Moon and the planets.
\end{abstract}

Key words: VLBI, spacecraft, narrow bandwidth, group delay, SELENE.

\section{Introduction}

The lunar gravity field has been investigated by Doppler measurement for the last 40 years. Recently its global mapping has been done by "Clementine" (Zuber et al., 1994) and "Lunar Prospector" (LP) (Konopliv et al., 2001). Although these observations contributed to knowing the low degree gravity field and the detail structure of the nearside, the gravity field of the rim of the Moon was not clearly investigated like the nearside. This is because the Doppler measurement is sensitive to the direction of the line of sight (LOS). Over the rim of the Moon, the gravity force from the Moon acts mainly in the direction perpendicular to LOS and we cannot estimate the gravity field precisely only by Doppler measurements. The internal structure, which is conventionally derived from the gravity and the topographic data (Bratt et al., 1985), is interesting especially for the rim of the moon. For example, the observational fact that a crust of the far side is older and thicker than that of the nearside is one of the important phenomena related to the thermal evolution of the moon, and there may be new facts under the rim region of the Moon which give us a key to clear up the cause of the dichotomy. In order to improve the low order and degree components of gravity field especially for

Copy right(c) The Society of Geomagnetism and Earth, Planetary and Space Sciences (SGEPSS); The Seismological Society of Japan; The Volcanological Society of Japan; The Geodetic Society of Japan; The Japanese Society for Planetary Sciences; TERRAPUB the rim of the Moon, we apply very long baseline interferometry (VLBI) technique in VRAD (the differential VLBI radio sources) mission of Japanese lunar exploration project SELENE (SELenological and ENgineering Explorer) in addition to a conventional 2-way Doppler and newly applied 4-way Doppler measurement (e.g., Hanada et al., 2002; Namiki et al., 1999). By measuring the difference of arriving time of the same wave front of a radio signal from the spacecraft (s/c) at two or more separated antennas, VLBI can precisely determine the position and the velocity of the $\mathrm{s} / \mathrm{c}$ in the direction perpendicular to LOS in contrast to the Doppler measurement.

The VLBI technique has been applied to tracking of a s/c since 1960s (e.g. Border et al., 1992; Sagdeyev et al., 1992). These VLBI observations of the s/c were successful, and recently it has been used for deep space missions of NASA and ESA (Antreasian et al., 2002; Thornton and Border 2003). However, a large amount of VLBI data prevented the real time processing. Moreover, frequency allocation of the downlink signals was not appropriate for precise group delay measurement and limited the accuracy of angular component of the position of the s/c to several tens to nano radians (Thornton and Border 2003). In order to detect a small motion of an s/c generated by the regional gravitational acceleration of the Moon, multi-frequency VLBI (MFV) was proposed (Kono et al., 2003). In MFV method, radio transmitters on board two small sub-satellites around 
Table 1. The characteristics of the narrow bandwidth VLBI sampling and recording system S-RTP station.

\begin{tabular}{ccc}
\hline Sampling rate & Quantization & Number of channels \\
\hline $200 \mathrm{kHz}$ & 6 bits & 4 \\
\hline
\end{tabular}

the Moon emit three carrier waves in $\mathrm{S}$ band and one in $\mathrm{X}$ band, and differential VLBI observation between the two sub-satellites is carried out. When we derived the fringe phase with an accuracy of 10 degrees by using differential VLBI, the relative position of two sub-satellites around the moon will be decided with an accuracy of $20 \mathrm{~cm}$ for the $2000 \mathrm{~km}$ baseline (Kono et al., 2003). This accuracy will be contributed on determination of the low degree coefficients of the spherical harmonics of the lunar gravity field (Matsumoto et al., 2002). In association with this plan, we have developed a narrow bandwidth sampling and recording system for VLBI and a correlation software for processing of the data obtained in the VRAD mission.

Using the systems mentioned above, VLBI observations for tracking of LP were carried out (Kono et al., 2003). In these observations, a phase delay was estimated within an error of a few degrees for a short period, however a group delay could not be estimated because only one frequency signal was used in LP. For applying VLBI to the gravity measurement of the Moon, we must obtain the phase delay for longer period within an error of a few degrees and determine the group delay without cycle ambiguity. In order to confirm the performance and capability of our narrow bandwidth VLBI system and analysis software, we conducted a fringe phase tracking and group delay determination for a few hours by VLBI observation of Japanese explorer Nozomi, which transmitted a main carrier wave and two sub carriers with frequencies of $515 \mathrm{kHz}$ apart from that of the main carrier. In this article, a detail of the VRAD system, and the result of the fringe phase tracking and the group delay measurement of Nozomi are shown.

\section{VRAD System}

\subsection{Back-end system}

In radio astronomical and geodetic VLBI surveys, usually wide bandwidth signals (from several $\mathrm{MHz}$ to $\mathrm{GHz}$ ) from radio stars have been recorded by a fast samplingrate system. This is because the signal-to-noise ratio (SNR) and the accuracy of the group delay depend on signal bandwidth. However, the wide bandwidth signals are not effective for real-time tracking of a s/c because they consume higher power in the $\mathrm{s} / \mathrm{c}$ and they need longer time to obtain a result from the large amount of data. For these reasons, we have developed a narrow bandwidth sampling and recording system for VLBI, called S-RTP station, for an s/c VLBI tracking. The performances of this system are listed in Table 1 and block diagram of this system is shown in Fig. 1. After the frequency down conversion and the video conversion of the signals by using K4 or Mark-4 systems, the S-RTP station samples and digitizes the video signals, and now a hard disk drive is used for recording sampled data instead of the tape recorder in the old system. Moreover, the phase calibration signals at every several tens of $\mathrm{kHz}$,

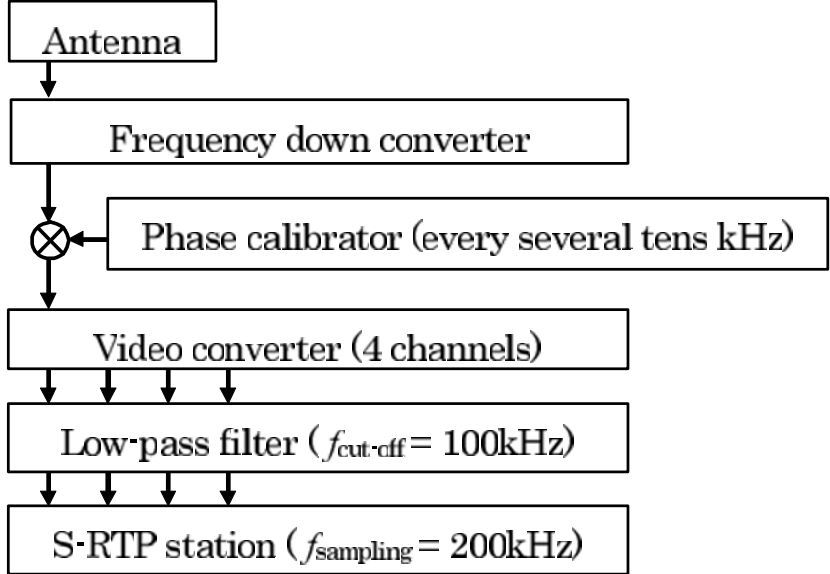

Fig. 1. Block diagram of the narrow bandwidth receiving system.

which are ajusted to the bandwidth of the S-RTP, are mixed to the signals to compensate the phase difference between channels in front of the video converter.

\subsection{Correlation software}

A correlation software has been also developed for this system. The software is composed of modules of VLBI delay estimation and cross-correlation which includes a bitshift, a fringe-stopping and a fractional bit correction.

2.2.1 Delay model for a $\mathbf{s} / \mathrm{c}$ in finite distance In a conventional correlator, a geometric delay is calculated assuming plane waves coming from the extra galactic radio sources. However, the assumption of the plane wave is not valid in the case of a s/c at a finite distance from the Earth. So we modified the delay model from the plane wave to a spherical wave to estimate the geometric delay for the s/c. In our method, the propagation times from the s/c to each ground station are calculated directly by using the light time equation. The propagation time from the $\mathrm{s} / \mathrm{c}$ to the reference station is expressed as follows,

$$
\tau_{r e f}(t)=\frac{\left|\boldsymbol{R}_{s c}\left(t-\tau_{r e f}(t)\right)-\boldsymbol{R}_{r e f}(t)\right|}{c}+\tau_{g r a v}
$$

where $\boldsymbol{R}_{s c}(t)$ is the predicted position vector of the s/c and $\boldsymbol{R}_{r e f}(t)$ represents the position vector of the reference station. $t$ is the time based on the reference station, $c$ is the velocity of light, and $\tau_{\text {grav }}$ is general relativistic delay. We obtain $\tau_{r e f}(t)$ from an iterative procedure as follows,

$$
\begin{array}{r}
\tau_{r e f}^{i+1}(t)=\frac{\left|\boldsymbol{R}_{s c}\left(t-\tau_{r e f}^{i}(t)\right)-\boldsymbol{R}_{r e f}(t)\right|}{c}+\tau_{g r a v} \\
\tau_{r e f}^{0}(t)=\frac{\left|\boldsymbol{R}_{s c}(t)-\boldsymbol{R}_{r e f}(t)\right|}{c}+\tau_{\text {grav }}
\end{array}
$$

$\tau_{r e f}^{0}(t)$ is an initial value of $\tau_{r e f}(t)$. On the other hand, the propagation time from the $\mathrm{s} / \mathrm{c}$ to the slave station is expressed as follows,

$$
\begin{aligned}
\tau_{s l v}(t)= & \frac{\left|\boldsymbol{R}_{s c}\left(t-\tau_{r e f}(t)\right)-\boldsymbol{R}_{s l v}\left(t-\tau_{r e f}(t)+\tau_{s l v}(t)\right)\right|}{c} \\
& +\tau_{\text {grav }}
\end{aligned}
$$

where $\boldsymbol{R}_{s l v}(t)$ is the position vector of the slave station and $\tau_{r e f}(t)$ is given from the Eq. (1). $\tau_{s l v}(t)$ can be obtained 
Table 2. List of radio telescopes involved in the VLBI observation of Planet-B.

\begin{tabular}{ccccc}
\hline Station & Antenna diameter $(\mathrm{m})$ & System noize temperature $(\mathrm{K})$ & Longitude & Latitude \\
\hline USUDA & 64 & 70 & $138^{\circ} 21^{\prime} 57^{\prime \prime} \mathrm{E}$ & $36^{\circ} 07^{\prime} 45^{\prime \prime} \mathrm{N}$ \\
KASHIMA & 34 & 85 & $140^{\circ} 39^{\prime} 36^{\prime \prime} \mathrm{E}$ & $35^{\circ} 57^{\prime} 06^{\prime \prime} \mathrm{N}$ \\
MIZUSAWA & 10 & 130 & $141^{\circ} 07^{\prime} 56^{\prime \prime} \mathrm{E}$ & $39^{\circ} 08^{\prime} 00^{\prime \prime} \mathrm{N}$ \\
MIZUSAWA & 20 & 150 & $141^{\circ} 07^{\prime} 57^{\prime \prime} \mathrm{E}$ & $39^{\circ} 08^{\prime} 01^{\prime \prime} \mathrm{N}$ \\
\hline
\end{tabular}

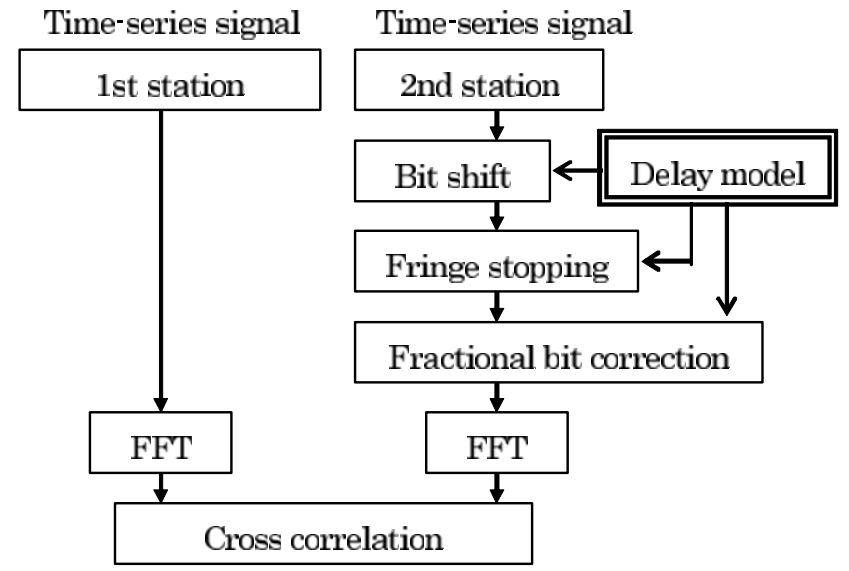

Fig. 2. Block diagram of the correlation process of the software.

from an iterative procedure in the same way as $\tau_{\text {ref }}(t)$. Finally the geometric delay between the reference and the slave station is obtained as follows,

$$
\tau_{\text {geometric }}(t)=\tau_{s l v}(t)-\tau_{r e f}(t)
$$

2.2.2 Correlation process The correlation process is made by FX mode. The signal recorded at the 1st station is Fourier transformed for every parameter period (PP). On the other hand, the signal recorded at the 2nd station is bit-shifted, fringe-stopped and corrected for the fractional bit by using the predicted geometric delay calculated from Eq. (5). After these corrections, the signal of the 2nd station is Fourier transformed. Finally, each Fourier component is cross-correlated and a residual fringe phase is obtained. Block diagram of the process is shown in Fig. 2. In many cases, the downlink signal from a s/c is a narrow bandwidth carrier wave. So, we use only several tens of $\mathrm{Hz}$ around the center frequency of the carrier wave to obtain a high SNR in the course of calculating the residual fringe phase.

\section{VLBI Observation of Nozomi}

Nozomi is the Japanese explorer that had two swingbys by the earth in December 2002 and June 2003 so as to be injected into a Mars orbit in 2004 (Yoshikawa et al., 2001). During the period between these two swingbys, the onboard high gain antenna could not point to the Earth due to some operational problems. In this period, range and Doppler measurements could not be conducted with sufficient SNR. To overcome this problem, the Nozomi team of JAXA (Japan Aerospace Exploration Agency) and the VLBI group of NICT (National Institute of In- formation and Communications Technology) collaborated to determine the Nozomi orbit by using the VLBI technique. We also took part in the observation from May 13 to 27,2003 , by using our VLBI systems. The radio telescopes involved in this observation were USUDA (USD), KASHIMA (KSM), and MIZUSAWA (MZS). Details of these telescopes are shown in Table 2.

Usually the differential VLBI observation between an s/c and a QSO is carried out to remove the fluctuations in fringe phase and propagation delay caused by ionosphere, troposphere, and the ground systems. However, the switching observation was not applied in our observation to detect a long-term behavior of fringe phase. Several QSOs with positions well known, for example $1842+681$ and $1849+670$, were observed before and after the tracking of Nozomi for calibrating the clock offset and the clock rate between VLBI stations. $\mathrm{X}$ band signals from QSOs were recorded in 4 channels of the $\mathrm{K} 5$ system, in which the sampling rate and quantization were $4 \mathrm{MHz}$ and 2 bit, respectively (Kondo et al., 2002).

\subsection{Signals of Nozomi}

Between the two swing-by events, the downlink signal of Nozomi was either in telemetry mode or in ranging mode. There was only one carrier wave in the telemetry mode. In contrast, the downlink signal consists of a main carrier wave, two range tones, and some ambiguity tones in the ranging mode. We observed Nozomi in ranging mode to estimate the group delay. The frequency of the main carrier wave $f_{\text {carrier }}$ was $8411 \mathrm{MHz}$ and those of the two range tones were $f_{\text {carrier }}+/-f_{\text {carrier }} / 2^{14}\left(f_{\text {carrier }} / 2^{14}\right.$ is about $515 \mathrm{kHz}$ ). The ambiguity tones with frequencies of $f_{\text {carrier }}+/-f_{\text {carrier }} / 2^{n+14}(n=1,2$. . ) were added every 100 seconds for 40 seconds to solve the ambiguity of the range tones. However, the carrier to noise ratios $(\mathrm{C} / \mathrm{N})$ of the ambiguity tones were so weak that these tones could not be detected. The main carrier wave and the two range tones were separately recorded in three channels of S-RTP station. To compensate the phase difference between these three channels, the phase calibration signals at every $60 \mathrm{kHz}$ were mixed with the IF signals in front of the video converter.

\subsection{Result of correlation}

3.2.1 Clock offset and clock rate The correlation of the signal from QSO was also carried out by the same software. Correlation process of the QSO was similar to the conventional method and the delay model was the same as the Consensus model (McCarthy, 2003). As a result of the correlation and the group delay analysis, the clock offset and the clock rate were estimated within an error of 20 pico seconds (psec) and of $0.001 \mathrm{psec} / \mathrm{second}$, respectively. 


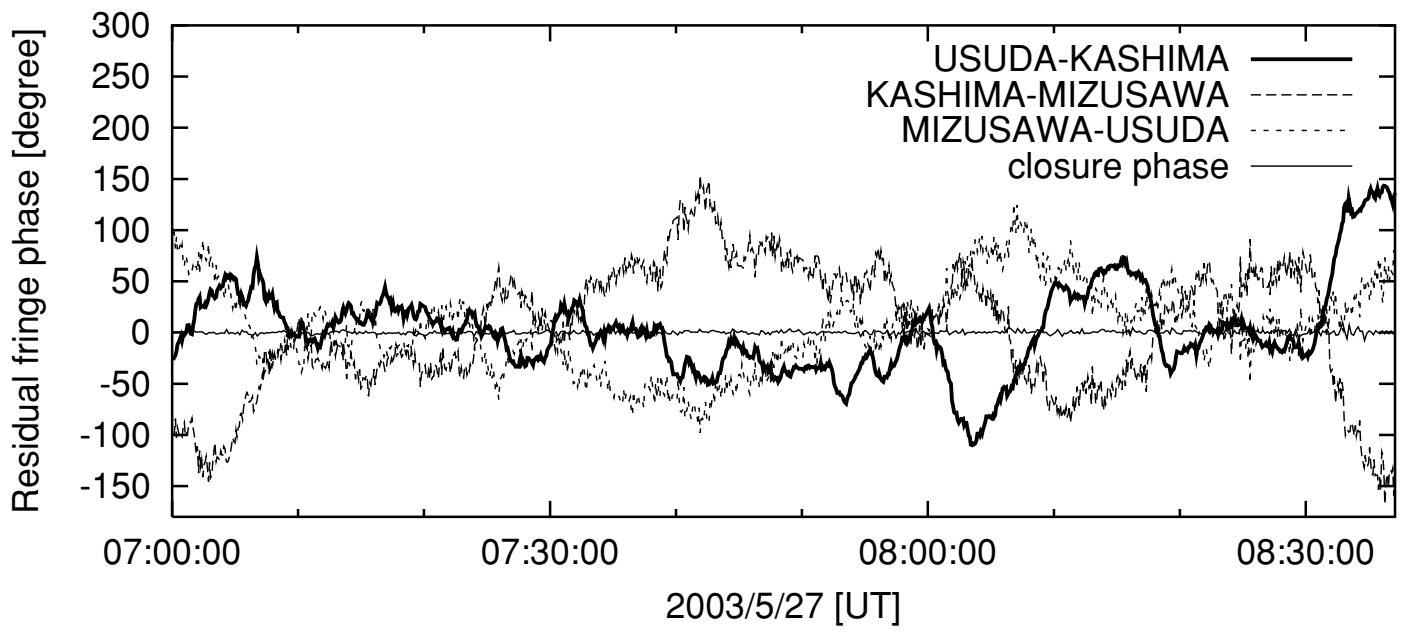

Fig. 4. The residual fringe phases of the main carrier wave for 3 baselines and the closure phases.

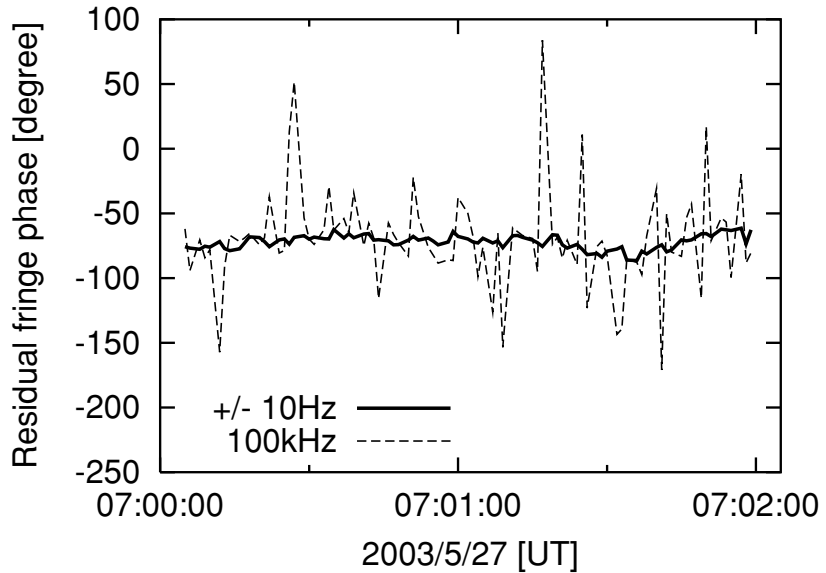

Fig. 3. The residual fringe phases of the main carrier wave for USD-KSM baseline.

These clock parameters were corrected before the correlation of the signals from Nozomi.

3.2.2 Estimation of residual fringe phase The frequency of the carrier waves from Nozomi changed slightly by a few $\mathrm{Hz}$ in $1 \mathrm{PP}$ of 1.3 seconds. So the Fourier components of only $+/-10 \mathrm{~Hz}$ around the center frequency of the carrier wave were used to calculate the residual fringe phase with a high SNR. The frequency resolution was $0.76 \mathrm{~Hz}$ for $1 \mathrm{PP}$. The residual fringe phases for USD-KSM baseline calculated by using the whole recorded bandwidth and only $+/-10 \mathrm{~Hz}$ around the center frequency of the main carrier wave are shown in Fig. 3. The root mean square (RMS) of the residual fringe phase for the period of 120 seconds decreased from 39 to 8 degrees by this method. The averaged SNR of the main carrier wave is about 40 for 40 seconds integration.

Moreover, we could continuously track the residual fringe phases of the main carrier wave for most observations of all baselines, i.e. USD-KSM, KSM-MZS, and MZS-USD. The residual fringe phases of the main carrier waves in each baseline from 07:00:00 to 08:40:00 on May 27, 2003 in UT and the closure phases are shown in Fig. 4.
The RMS of the closure phases is 3 degrees for the period of 100 minutes when the integration time of the residual fringe phases are 5.2 seconds. This result suggests that the correlation by the software method has been carried out successfully, and the intrinsic error of the residual fringe phase, which is caused by the thermal noise of the ground systems or the model of the software, is 3 degrees. Furthermore, the residual fringe phase of each baseline varies with various periods from several tens of seconds through several tens of minutes as shown in Fig. 4. To clarify these fluctuations, the Allan standard deviation (ASD) of the residual fringe phases were calculated for each baseline and are shown in Fig. 5. From Fig. 5, the ASD decreased at the rate of $1 / \tau$ ( $\tau$ is the averaging time) until $\tau$ was less than 10 seconds for USD-KSM and 30 seconds for KSM-MZS and MZSUSD. Consequently, the short-term variation of the residual fringe phase would be explained by the thermal noise of the ground systems. The difference of the ASD for three baselines would be attributed to the difference of the parameters of the ground systems: system temperature, diameter of the antenna, coherence factor, and aperture efficiency of the antenna. The ratios of the ASD expected from the nominal value of the parameters of the ground systems are 11 for USD-KSM/KSM-MZS, 5 for USD-KSM/MZS-USD, and 3 for MZS-USD/KSM-MZS. These values are roughly consistent with Fig. 5. While $\tau$ is longer than 10 seconds for USD-KSM and 30 seconds for KSM-MZS and MZS-USD, the fluctuation of the atmosphere became dominant and the ASD hardly decreased. The ASD of the residual fringe phases caused by the atmospheric fluctuation varies from $10^{-12}$ to $10^{-13}$ according to the atmospheric condition, and $1.5 \times 10^{-13}$ from Fig. 5 corresponds to the value of clear day. In addition, the ASD for longer than 200 seconds was not calculated. This is because the observation period of about 6,000 seconds is not enough to obtain the ASD for longer than 200 seconds.

Two range tones were correlated by the same way as the main carrier wave to carry out the group delay analysis. In contrast to the main carrier wave, we could detect the residual fringe phase of the range tones only for the USDKSM baseline because their SNR was less than that of the 


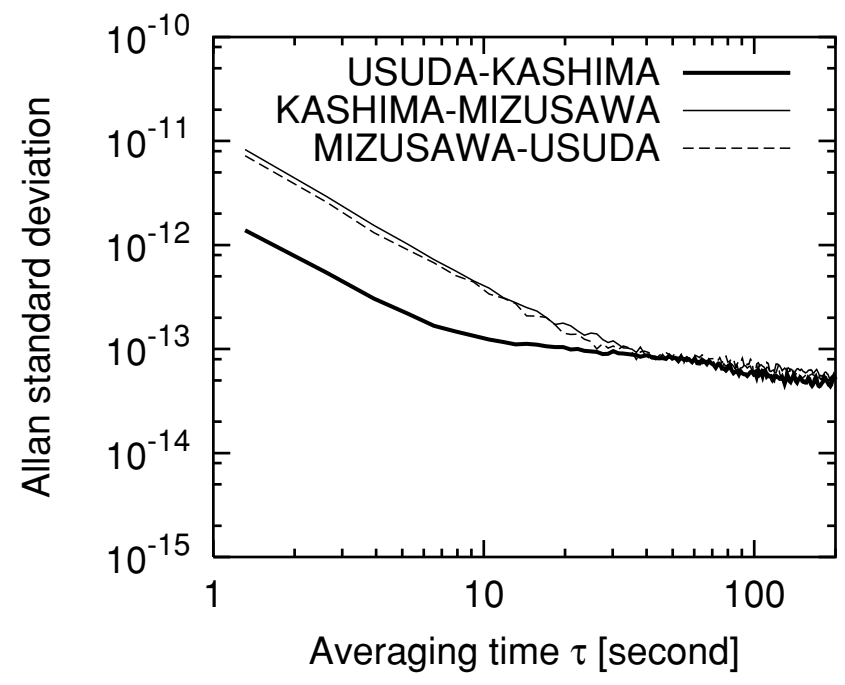

Fig. 5. The Allan standard deviations of residual fringe phases of the main carrier wave for 3 baselines.

main carrier wave. Moreover, the transmission of Nozomi in ranging mode was divided into 2 modes, $\mathrm{A}$ and $\mathrm{B}$. In the mode $\mathrm{A}$, the ambiguity tones were added to the downlink signal and decreased the SNR of the range tones. So the residual fringe phases of the range tones were not obtained for this period of 60 seconds as shown in Fig. 6. On the other hand, the ambiguity tones were not added in the mode $\mathrm{B}$ and the residual fringe phases of the range tones were obtained in this period of 40 seconds in B of Fig. 6. The averaged SNR of two range tones in the mode B is 19 for 40 seconds integration. Therefore the group delay analysis could only be carried out during the mode B.

3.2.3 Estimation of group delay Before analyzing the group delay, the phase difference of the separate channels obtained from the phase calibrator signals has to be removed from the residual fringe phase as follows,

$$
\delta \phi_{i}^{\prime}=\delta \phi_{i}-\left(\phi_{p c a l, i}^{r e f}-\phi_{p c a l, i}^{s l v}\right),(i=1,2,3)
$$

where $\delta \phi_{i}$ is the residual fringe phase of $i$ th channel and $\phi_{\text {pcal }, i}^{\text {ref }}$ and $\phi_{\text {pcal }, i}^{s l v}$ are the phase of the phase calibration signal in $i$ th channel, respectively. The residual group delay of the signals is given by,

$$
\delta \tau_{\text {group-delay }}=\frac{\delta \phi_{i+1}^{\prime}-\delta \phi_{i}^{\prime}}{2 \pi\left(f_{i+1}-f_{i}\right)}
$$

where $f_{i}$ is the frequency of the carrier wave. The propagation delays of the troposphere and the ionosphere are also corrected. The atmospheric delay is corrected by using the tropospheric model (Davis et al., 1985) and the ionospheric delay is corrected by using a regional ionosphere map (Ping et al., 2002).

After these corrections, the geometric delays of the signals from Nozomi are obtained in every 100 seconds by the group delay analysis. These derived geometric delays well accorded with the geometric delay determined by the range and Doppler measurements, and the differences between these geometric delays are shown in Fig. 7. The average of these differences are about 2 nano seconds (nsec) and this result showed that the group delay is obtained without cycle ambiguity of about 2 micro seconds, which is the inverse of the frequency intervals of the three carrier waves. On the contrary, the RMS of the differences between the geometric delays is $13 \mathrm{nsec}$. The tropospheric and the ionospheric fluctuations were at the order of a few nsec during the observation period, so most of the error of the group delay could be caused by the thermal noise in the ground system. The SNR of the two range tones are almost half of that of the main carrier wave, which is 19 for 40 seconds integration in average. The error of the group delay expected from the SNR of the range tone is $12 \mathrm{nsec}$ and is in consistency with the RMS of the differences of the geometric delays. The baseline length is about $200 \mathrm{~km}$ for USD-KSM baseline, the precision of the angular distance of Nozomi is 3710 mas. The distance between Nozomi and the ground station is $6 \times 10^{6} \mathrm{~km}$ in this observation, so the estimated position error of Nozomi is $117 \mathrm{~km}$. Moreover, it could be expected that the accuracy of the angular distance and the position of Nozomi would be improved to 46 mas and 1.4 $\mathrm{km}$ respectively, if the SNR of the range tones are as large as the main carrier wave and the frequency intervals are 20 $\mathrm{MHz}$.

\section{Discussion}

As mentioned in the previous section, we can continuously track the residual fringe phase of the main carrier wave for 100 minutes and obtain the group delay of the three carrier waves without cycle ambiguity. These results confirm the validity of the narrow bandwidth hardware and software VLBI systems. In this section, we suppose the feasibility of the VRAD mission of SELENE from the result of Nozomi considering the $\mathrm{C} / \mathrm{N}$ of each observation.

The three carrier waves in S-band whose frequency intervals are $6 \mathrm{MHz}$ and $75 \mathrm{MHz}$ will be received to obtain the precise group delay on differential VLBI observation of two sub-satellites in VRAD. In order to obtain the group delay without the cycle ambiguity, the phase error of the residual fringe phases of these carrier waves are required to be less than 10 degrees between the switching period of $120 \mathrm{sec}-$ onds. In the case of the main carrier wave of Nozomi for the baseline of USD-KSM, the C/N is 6 and the RMS of the residual fringe phases of every $1 \mathrm{PP}$ of 1.3 seconds during 120 seconds is 8 degrees. In contrast, the specification of the $\mathrm{C} / \mathrm{N}$ of the carrier waves in VRAD is 3.5 , and the phase error is expected to be 14 degrees for $1 \mathrm{PP}$ of 1.3 second in VRAD because the phase error would be proportional to the inverse of the $\mathrm{C} / \mathrm{N}$. This phase error would be improved by the square root of the integration period. By integrating the residual fringe phases for $3 \mathrm{PP}$, the phase error would be reduced to 6 degrees and the requirement of 10 degrees in VRAD would be achieved. This result suggests that the group delay of the three carrier waves would be obtained without cycle ambiguity and the small acceleration of the sub-satellite caused by the gravity field of the Moon will be detected from the geometric delay.

\section{Conclusion}

We carried out a VLBI observation of Nozomi. In this observation, we recorded three carrier wave signals by us- 


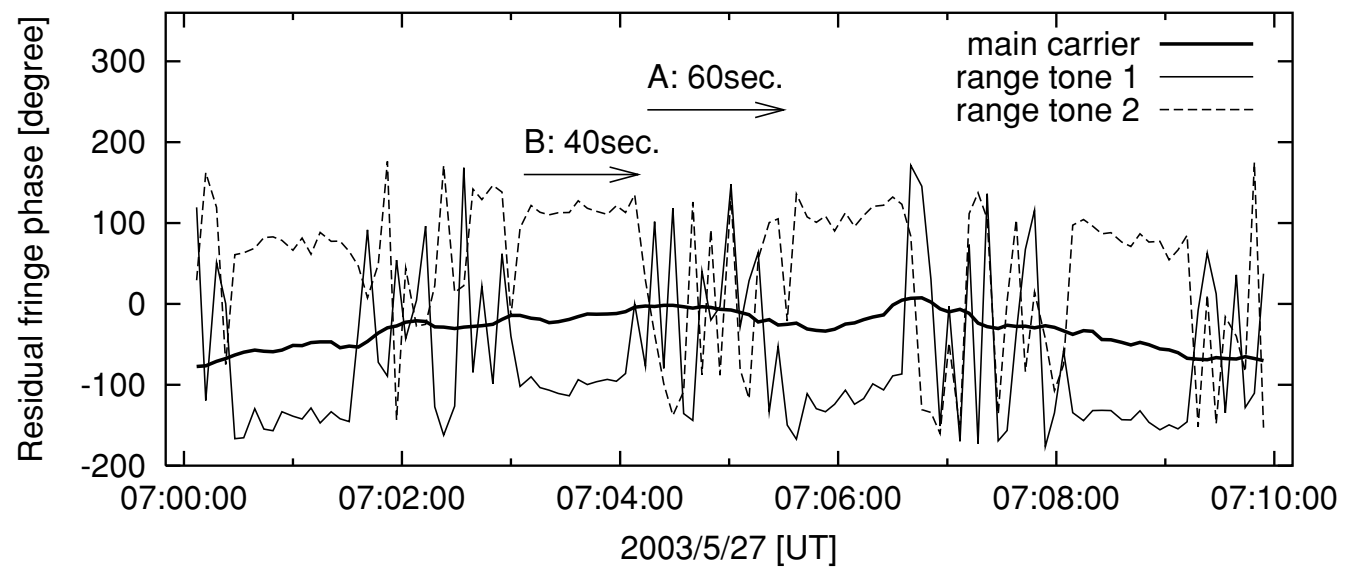

Fig. 6. The residual fringe phases of the main carrier wave and two range tones for USD-KSM baseline.

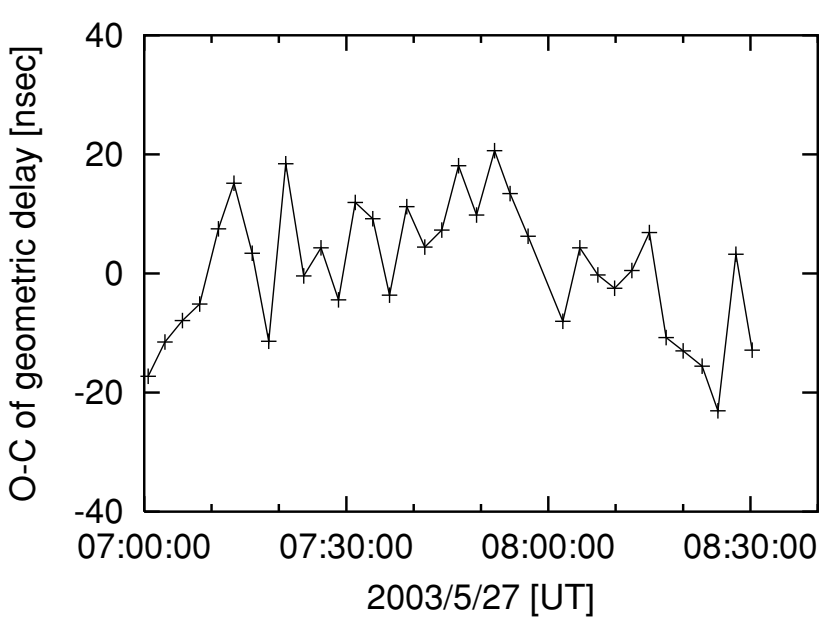

Fig. 7. The difference between the geometric delays in this VLBI observation and those calculated from the orbital motion estimated from the range and Doppler measurements.

ing dedicated narrow bandwidth VLBI system, and correlated these signals by the software method. As a result of correlation, the residual fringe phases of the main carrier wave in each baseline are obtained at every 1.3 seconds. We can also continuously track them for 100 minutes. The variation of the residual fringe phase is $+/-150$ degrees in peak-to-peak and its Allan standard deviation shows that the variation comes from the fluctuation of the atmosphere. Moreover, we can derive successfully the group delays for every 100 seconds by using three carrier wave signals. The RMS of them is 13 nsec. The average of the obtained geometric delay well accorded with the geometric delay determined by the range and Doppler measurement within an error of 2 nsec. From these results, it could be expected that if the SNR of the range tones are as large as the main carrier wave and the frequency intervals are $20 \mathrm{MHz}$, the accuracy of the angular distance and the position of Nozomi would be improved to be less than 46 mas and $1.4 \mathrm{~km}$ respectively.

From the result of the correlation and the group delay analysis, we confirmed the validity of the new hardware and the software in VLBI system. We also confirmed the feasibility of the VRAD mission of SELENE. That is, the phase error would be less than 10 degrees for 3.9 seconds integration, which is the requirement of VRAD. It could be expected that we can detect the regional gravity field of the Moon precisely by using this system. Furthermore, in addition to the range and Doppler measurements, VLBI observation by this system has a capability of precise threedimensional positioning of an s/c.

Acknowledgments. We would like to thank all the staffs of Kashima space communication research center of NICT, Usuda deep space center of JAXA and Mizusawa observation center of National Astronomical Observatory for their helps in VLBI observations.

\section{References}

Antreasian, P. G., D. T. Baird, J. S. Border, P. D. Burkhart, E. J. Graat, M. K. Jah, R. A. Mase, T. P. McElrath, and B. M. Portock, 2001 Mars Odyssey Orbit Determination During Interplanetary Cruise, AIAA/AAS Astrodynamics Specialist Conference and Exhibit, AIAA-2002-4531, Aug. 5-8, 2002.

Border, J. S., W. M. Folkner, R. D. Kahn, and K. S. Zukor, Precise Tracking of the Magellan and Pioneer Venus Orbiters by Same-Beam Interferometry, Part 1: Data Accuracy Analysis, TDA progress report, 42-110, Aug. 15, 1992.

Bratt, S. R., S. C. Solomon, J. W. Head, and C. H. Thurber, The deep structure of lunar basin: Implications for basin formation and modification, J. Geophys. Res., 90, 3049-3064, 1985.

Davis, J. L., T. A. Herring, I. I. Shapiro, A. E. E. Rogers, and G. Elgered, Geodesy by radio interferometry: Effects of atmospheric modeling errors on estimates of baseline length, Radio Sci., 20, 1593-1607, 1985.

Hanada, H., T. Iwata, Y. Kono, and K. Matsumoto, VRAD Mission: Precise Observation of Orbits of Sub-satellites in SELENE, IVS 2002 General Meeting Proceedings, 73-76, 2002.

Kondo, T., Y. Koyama, J. Nakajima, M. Sekido, R. Ichikawa, E. Kawai, H. Okubo, H. Osaki, M. Kimura, Y. Ichikawa, and GALAXY team, Real-time Gigabit VLBI System and Internet VLBI System, IVS 2002 General Meeting Proceedings, 142-146, 2002.

Kono, Y., H. Hanada, J. S. Ping, Y. Koyama, Y. Fukuzaki, and N. Kawano, Precise positioning of spacecrafts by multi-frequency VLBI, Earth Planets Space, 55, 581-589, 2003.

Konopliv, A. S., S. W. Asmer, E. Carranza, W. L. Sjogren, and D. N. Yuan, Recent gravity models as a result of the Lunar Prospector Mission, Icarus, 150, 1-8, 2001.

Matsumoto, K., K. Heki, and H. Hanada, Global Lunar Gravity Field Recovery from SELENE, IVS 2002 General Meeting Proceedings, 381385, 2002.

McCarthy, D. D., IERS Conventions 2003, in IERS Technical Note 32, to be published.

Namiki, N., H. Hanada, T. Tsubokawa, N. Kawano, M. Ooe, K. Heki, T. Iwata, M. Ogawa, and T. Takano, Selenodetic experiments of SELENE: Relay satellite, differential VLBI, and laser altimater, Adv. Space Res., 
23, 1817-1820, 1999.

Ping, J. S., Y. Kono, K. Matsumoto, Y. Otsuka, A. Saito, C. Shum, K. Heki, and N. Kawano, Regional ionosphere map over Japanese Islands, Earth Planets Space, 54, e13-e16, 2002.

Sagdeyev, R. Z., V. V. Kerzhanovitch, L. R. Kogan, V. I. Kostenko, V. M. Linkin, L. I. Matveyenko, R. R. Nazirov, S. V. Pogrebenko, I. A. Struckov, R. A. Preston, J. Purcel, C. E. Hildebrand, V. A. Grishmanovskiy, A. N. Kozlov, E. P. Molotov, J. E. Blamont, L. Boloh, G. Laurans, P. Kaufmann, J. Galt, F. Biraud, A. Boischot, A. OrtegaMolina, C. Rosolen, G. Petit, P. G. Mezger, R. Schwartz, B. O. Ronnang, R. E. Spencer, G. Nicolson, A. E. E. Rogers, M. H. Cohen, R. M. Martiroysan, I. G. Moiseyev, and J. S. Jatskiv, Differential VLBI measurements of the Venus atmosphere dynamics by balloons: VEGA project, Astron. and Astrophys., 254, 387-392, 1992.

Thornton, C. L. and J. S. Border, Radiometric Tracking Techniques for Deep Space Navigation, JPL Deep Space Communication and Naviga- tion Series, edited by J. H. Yuen, 85 pp, Wiley and Sons Inc., US, 2003. Yoshikawa, M., M. Sekido, N. Kawaguchi, K. Fujisawa, H. Hanada, Y. Kono, H. Hirabayashi, Y. Murata, S. Sawada-satoh, K. Wajima, Y. Asaki, J. Kawaguchi, H. Yamakawa, T. Kato, T. Ichikawa, and T. Ohnishi, Present status and future problems of the orbit determination for Nozomi spacecraft, IVS CRL Technology Development Center News, No. 19, 37-40, 2001.

Zuber, M. T., D. E. Smith, F. G. Lemoine, and G. A. Neumann, The shape and internal structure of the Moon from Clementine Mission, Science, 266, 1839-1843, 1994.

F. Kikuchi (e-mail: fuyuhiko@miz.nao.ac.jp), Y. Kono, M. Yoshikawa, M. Sekido, M. Ohnishi, Y. Murata, J. Ping, Q. Liu, K. Matsumoto, K. Asari, S. Tsuruta, H. Hanada, and N. Kawano 